\title{
COMPARAISON ENTRE L'AGE ET LA CROISSANCE DÉTERMINÉS PAR SCALIMÉTRIE ET OTOLIMÉTRIE CHEZ LA TRUITE DE MER (SALMO TRUTTA L.)
}

\author{
J.L. BAGLINIERE (1), G. LECLERC (1), A. RICHARD (2)
}

(1) Laboratoire d'Ecologie Hydrobiologique - INRA, 65 Route de St Brieuc, 35042 RENNES CEDEX, France.

(2) Délégation Régionale du Conseil Supérieur de la Pêche, 84 rue de Rennes, 35510 CESSON SEVIGNE, France.

\section{RESUME}

La détermination de l'âge et de la croissance à partir des écailles et des otolithes (entiers et coupés) est comparée sur un échantillon de truite de mer (essentiellement $\mathrm{O}^{+}$de mer). Le pourcentage d'agrément entre les méthodes varie entre $93,5 \%$ et $98,9 \%$, cette valeur maximale étant obtenue entre les écailles et les otolithes entiers. Les longueurs rétromesurées à partir des otolithes sont significativement supérieures $(p<0,01)$ à celles estimées par scalimétrie. Différents phénomènes inscrits sur les écailles n'apparaissent pas sur les otolithes.

Les résultats sont discutés ; les avantages et les inconvénients des différentes méthodes sont comparés. II en ressort qu' une utilisation de l'otolithe, aussi rentable que celle de l'écaille chez la truite de mer, passe par une observation plus fine de la structure.

\section{ABSTRACT \\ COMPARISON OF AGE AND GROWTH DETERMINATION BY SCALE AND OTOLITH READING IN SEA TROUT (SALMO TRUTTA L.).}

Determination of age and growth from scales and otoliths (whole and sectioned) is compared on sea-trout sample (mainly finnock). Agreement between the methods varies from $93,5 \%$ to $98,9 \%$, the highest value being observed between scales and whole otoliths. Back-calculated length from otoliths are significatively $(p<0,01)$ greater than these from scales. Different phenomena visible on scales are not seen on otoliths.

Results are discussed; advantages and disadvantages of different methods are compared. From this it is concluded that otolith use as inexpensive as scale use requires the observation of otolith microstructure in sea trout.

\section{I - INTRODUCTION}

La détermination correcte de l'âge chez le poisson est nécessaire à la bonne connaissance du cycle biologique de l'espèce et de la dynamique de ses populations. Chez les salmonidés, la détermination de l'âge est souvent effectuée à partir des écailles car c'est, en général, une méthode fiable et d'utilisation simple (SYCH, 1967 ; JENSEN, 1968 ; MAC PHAIL, 1974 ; BAGLINIËRE, 1985). Cependant, leur interprétation peut différer fortement comme cela a été montré chez le saumon atlantique (PIPPY et REDDIN, 1982). Un souci d'homogénéisation a entraîné la réunion d'ateliers de lecture d'écailles international (SHEARER, com. pers. ) et national (BAGLINIËRE, 1985). De ces deux ateliers, il est ressorti la nécessité d'approfondir les recherches dans le domaine de la scalimétrie chez le saumon atlantique.

Chez la truite de mer, l'interprétation des écailles semble plus compliquée dans bien des cas (JARVI et MENZIES, 1936 ; BEALL, 1979; RICHARD, 1981 et données non publiées). Ainsi, il est apparu intéressant, pour cette espèce, de comparer la détermination de l'âge obtenu à partir des écailles avec celle effectuée à partir d'une structure osseuse, l'otolithe.

Ce travail a été réalisé sur le stock de truite de mer de la rivière Calonne (affluent de la Touques. Basse-Normandie) où une étude de caractérisation biologique et physiologique du stade $\mathrm{O}^{+}$de mer a été entreprise. Les résultats présentés concernant la comparaison de l'âge et de la croissance déter minés par scalimétrie et otolimétrie se rapportent presque exclusivement à cette classe d'âge de mer. 


\section{II - MATERIEL ET METHODES}

Les truites de mer ont été capturées par piégeage lors de leur remontée au mois de juillet et août 1985. Leur longueur fourche est comprise entre 230 è 400 millimètres, valeur limite supérieure de la taille des $0^{\prime}$ de mer (RICHARD, données non publiées). La prise d'écailles a été réalisée sur une zone standard (BAGLINIERE, 1985). Les écailles ont été imṕrimées sur un film plastique (PVC) de $1 \mathrm{~mm}$ d'épaisseur et lues à l'aide d'un projecteur macro (x 50). Des marques de fraie dans la partie eau douce ont pu être notées comme chez le saumon atlantique (Salmo salar) (MAISSE et BAGLINIĖRE, 1983 ; BAGLINIËRE et MAISSE, 1985). Les marques de fraie au "stade adulte correspondent aux structures observées par JARVI et MENZIES (1936); BACKIEL et SYCH (1958); BEALL (1979) et RICHARD (1981).

Les otolithes ont été prélevés au laboratoire et conservés dans de la glycérine. Comme pour la plupart des espèces, seule la sagitta a été utilisée (PANNELLA, 1971 ; EVERSON, 1980 ; CASSOULEINS et CASSOU-LEINS, 1981 ; DENIEL, 1981 ; RADTKE, 1984 ; BERG, 1985 ; HOYER et al., 1985). Les otolithes ont été préparés selon deux méthodes. Dans le premier cas, ils ont été inclus dans de la résine polyester noire, puis découpés en trois lames minces suivant une technique mise au point par SOUPLET et DUFOUR (1983) et dérivée de celle utilisée par BEDFORD (1980). Leur lecture a été faite en lumière réfléchie avec un projecteur micro (x 300). Dans le deuxième cas, les otolithes entiers. conservés dans de la glycérine durant deux mois, ont été lus directement à l'aide d'un projecteur macro en lumière réfléchie (x 30 ). Un otolithe est considéré comme illisible lorqu'aucun anneau n'apparaît distinctement sur la structure.

Des relations linéaires, longueur fourche - longueur écaille et longueur fourche - longueur de l'otolithe, ont été établies à partir d'un même échantillon d'écailles non régénérées et d'otolithes entiers lisibles. La longueur de l'écaille est mesurée le long de son plus grand axe. Celle de l'otolithe est définie par le rayon " $r$ " présenté en figure 1. Dans les deux cas les tailles rétromesurées $Y_{i}$ à la fin des hivers d'eau douce sont calculées à partir des relations trouvées de la manière suivante pour un modèle linéaire (LECREN, 1947) $y_{i}=y-b\left(X-X_{i}\right)$.

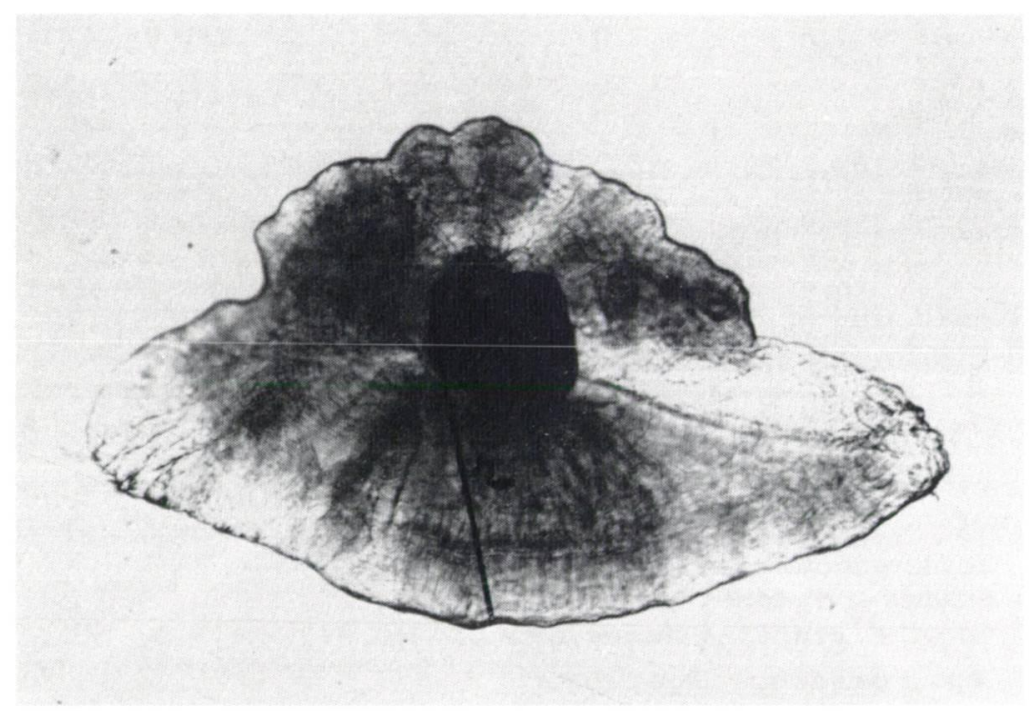

Figure 1: Forme générale d'un otolithe de truite de mer et présentation du rayon (r) utilisé pour la relation longueur du poisson - longueur de l'otolithe.

Figure 1: General shape of sea trout otolith and presentation of radius used in the relation fish length - otolith length.

\section{III - REsultats}

\section{1. - Détermination de l'âge}

\subsection{1. - Ecailles et coupes fines d'otolithes (Tableau 1A)}

131 poissons ont été examinés. Dans cet échantillon les écailles de deux individus (1,5\%) sont régénérées alors que les otolithes sont interprétables. Les otolithes de 11 autres $(8,4 \%)$ sont illisibles 
A

\begin{tabular}{|c|c|c|c|c|c|c|c|c|c|c|c|c|}
\hline $\begin{array}{l}\text { otolithes } \\
\text { sectiomé }\end{array}$ & $s$ & $1.0^{+}$ & $:$ & $2.0^{+}$ & : & $3.0^{+}$ & $: 2.0^{+}$ & $\mathrm{F} 0^{+}$ & : & $1.1^{+}$ & $:$ & I11isibles \\
\hline : & $:$ & & : & & $:$ & & : & & : & & $:$ & \\
\hline : Ecaill & : & & $:$ & & : & & : & & : & & : & \\
\hline 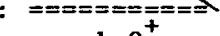 & $:$ & $=========$ & : & $== \pm=======$ & : & $==== \pm====$ & $: \quad====$ & $=====$ & : & $==x==0=0=$ & : & $===0===0=$ \\
\hline $1.0_{+}^{+}$ & : & 32 & : & 4 & : & - & $:$ & - & : & - & $:$ & 6 \\
\hline $2.0_{+}^{\top}$ & $:$ & 1 & : & 77 & : & - & $:$ & - & : & - & $:$ & 5 \\
\hline $3.0^{\top}+$ & : & - & : & - & : & 1 & : & - & : & - & : & - \\
\hline $2.0^{\circ} \mathrm{F}+\mathrm{O}^{\top}$ & $:$ & - & : & - & : & - & : & 0 & $:$ & 1 & $:$ & - \\
\hline $1.1^{\top}$ & : & - & : & - & : & - & : & - & : & 2 & $:$ & - \\
\hline regénérées & $:$ & - & : & 2 & : & - & $:$ & - & : & - & $:$ & 0 \\
\hline : & $:$ & & $\therefore$ & & $:$ & & $:$ & & $:$ & & $:$ & \\
\hline
\end{tabular}

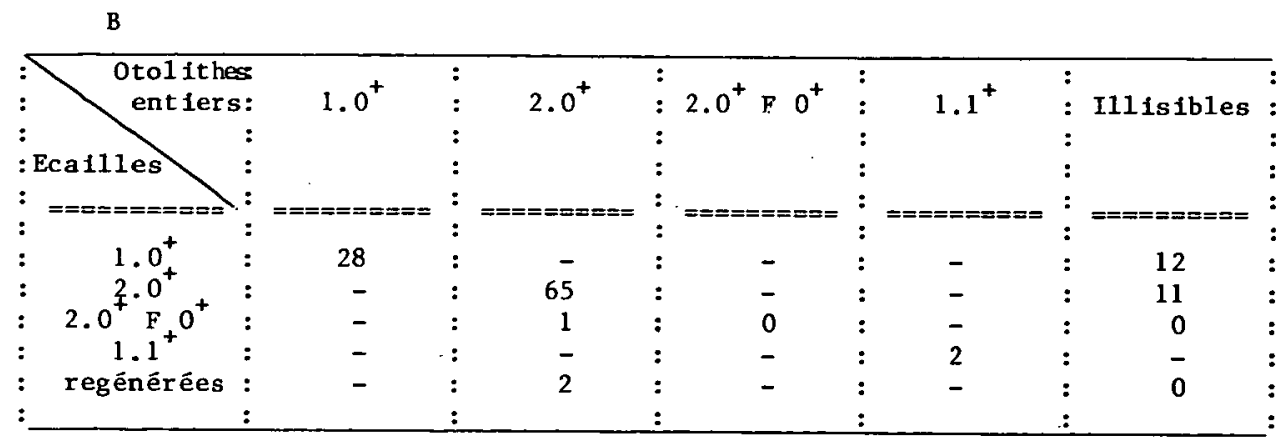

C

\begin{tabular}{|c|c|c|c|c|c|c|c|c|c|c|}
\hline $\begin{array}{l}\text { Otolithes } \\
\text { sectionné }\end{array}$ & & $1.0^{+}$ & : & $2.0^{+}$ & : & $1.1^{+}$ & : & $2.1^{+}$ & : & I111sibles \\
\hline & $:$ & & : & & $:$ & & : & & : & \\
\hline Otolithe & : & & : & & $:$ & & : & & : & \\
\hline entiers & & & : & & $:$ & & : & & : & \\
\hline 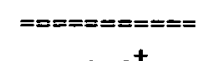 & $:$ & $======\therefore===$ & : & $====0====$ & $:$ & $=========$ & : & $\Rightarrow=\geq===\leq==$ & $\forall$ & $====0===$ \\
\hline $1.0_{+}^{+}$ & : & 20 & : & 4 & $:$ & - & $:$ & - & : & 2 \\
\hline $2.0+$ & $:$ & 1 & : & 64 & $:$ & - & : & 1 & : & 2 \\
\hline $1.1+$ & : & - & : & - & : & 2 & : & - & : & - \\
\hline 2.1 & $:$ & - & : & - & $:$ & - & : & 0 & : & - \\
\hline il1 isibles & $:$ & 11 & : & 10 & : & - & : & - & : & 2 \\
\hline & $:$ & & $:$ & & : & & : & & $:$ & \\
\hline
\end{tabular}

Tableau 1 : Comparaison de la détermination d'âge par scalimétrie et otolimétrie chez la truite de mer.
A - Ecailles et otolithes sectionnés (effectif 131)
B - Ecailles et otolithes entiers (effectif 121)
C - Otolithes coupés et entiers (effectif 119).
$2.0^{+}$âge d'eau douce - âge de mer
$F=$ fraie.

Table 1 : Comparison of age determination by scale and otolith reading in sea trout.
A - Scales and cutted otoliths (number 131)
B - Scales and whole otoliths (number 121)
C - Cutted and whole otoliths (number 119).
2.0 $0^{+}$freswater age - sea age
F = spawning.

alors que l'âge peut être déterminé à partir des écailles. Sur les 118 individus restants, le pourcentage d'agrément entre les deux méthodes est de 94,9\% laissant apparaître six cas de divergence. Parmi ceux-ci, cinq concernent l'âge d'eau douce et dans quatre cas l'otolithe surestime l'âge scalimétrique. Le dernier cas concerne l'âge de mer. Sur l'écaille, il apparaît une marque de fraie au stade $0^{+}$de mèr alors que sur l'otolithe au même stade seul un anneau hivernal est visible. Par ailleurs, en comparant les deux structures, on remarque que : 
- il n'est pas possible de définir la reprise de croissance du smolt sur l'otolithe alors qu'elle est présente sur $75 \%(24 / 32)$ des écailles de poisson âgés de 1 an d'eau douce et sur $27,3 \%$ de celles d'individus de 2 ans d'eau couce :

- la marque de fraie lors du dernier hiver est absente sur les otolithes d'individus de 2 ans d'eau douce alors qu'elle est notée sur $10,4 \%$ (8/77) des écailles de poisson de même âge ;

- la présence de "checks" ou faux anneaux est visible à la fois sur les écailles et les otolithes de $28.9 \%(9 / 32)$ individus d'un an d'eau douce.

\subsection{2. - Ecailles et otolithes entiers (Tableau 1B)}

121 poissons ont été examinés. Dans cet échantillon les écailles de deux individus ( $1,7 \%$ ) sont régénérées alors que les otolithes sont interprétables. Les otolithes de 23 autres (19\%) ne sont pas lisibles alors que l'âge peut être déterminé à partir des écailles. Sur les 96 individus restants le pourcentage d'agrément est de $\mathbf{9 8 , 9} \%$. Le seul cas de divergence concerne le poisson ayant frayé comme $\mathrm{O}^{+}$de mer puisque sur l'otolithe la marque de fraie n'apparaît pas et que le poisson est simplement âgé de 2 ans d'eau douce et de $\mathrm{O}^{+}$de mer. Sur l'otolithe entier n'apparaissent ni de reprise de croissance, ni de faux anneaux, ni de marque de fraie en eau douce.

\subsection{3. - Otolithes coupés et entiers (Tableau 1C)}

119 poissons ont été examinés. Les coupes fines de six otolithes (5\%) ne sont pas lisibles, alors que l'âge peut être déterminé d'après les otolithes entiers. Parmi ces derniers, 21 d'entre eux (17,6\%) ne sont pas interprétables alors que l'âge peut être déterminé à partir des sections d'otolithes. Sur les 92 individus comparés, le pourcentage d'agrément est de $93,5 \%$, six cas de divergence étant notés. Dans cinq d'entre eux, l'otolithe coupé surestime l'âge obtenu à partir de l'otolithe entier avec en particulier la présence d'un hiver de mer alors que cet anneau marin n'apparaît pas sur l'otolithe entier.

\section{2. - Comparaison de la croissance}

Les régressions linéaires entre la longueur du poisson et celles de l'écaille (1) ou de l'otolithe (2) sont calculées sur un échantillon de 100 individus. Ainsi l'on a :

$$
\begin{aligned}
& y=61,52+129,22 \times \quad r=0,91 \\
& \text { variance résiduelle }=265,45 \\
& y=\text { longueur du poisson }(\mathrm{mm}) \quad x=\text { longueur de l'écaille }
\end{aligned}
$$

$$
\begin{aligned}
& y=-12,38+268,78 \times \quad r=0,88 \\
& \text { variance résiduelle }=351,10 \\
& y=\text { longueur du poisson }(\mathrm{mm}) \quad x=\text { rayon de l'otolithe }
\end{aligned}
$$

Les tailles rétromesurées à partir des otolithes sont supérieures $(p<0,01)$ à celles estimées à partir des écailles pour les deux hivers d'eau douce (Tableau 2 et Figure 2). L'écart moyen des tailles rétromesurées par les deux méthodes n'est différent $(p<0,01)$ qu'entre les valeurs obtenues aux premier et second hivers des poissons de deux ans d'eau douce (Tableau 2). La variabilité de l'écart moyen apparaît beaucoup plus grande pour le premier hiver de la classe des deux ans d'eau douce (Figure 2).

\section{IV - DISCUSSION}

La comparaison de la détermination d’âge par scalimétrie et otolimétrie n'est pas réalisée fréquemment. Pour les quelques espèces étudiées apparaît dans l'ensemble un bon agrément entre les deux méthodes, sauf chez une population d'omble de fontaine (Sa/velinus fontinalis) du Québec et chez celle de truite commune (Salmo trutta) sédentaire du nord de la Norvège (Tableau 3). Dans ces deux cas l'otolithe apparait la structure la plus fiable pour l'estimation de l'âge en raison du faible taux de croissance (populations plus âgées) (JONSSON, 1976; DUTIL et POWER, 1977), et ceci dès l'apparition de la maturation (JONSSON, 1976). Dans le cas de la population de truite de mer de la Calonne où la croissance est plus élevée, les pourcentages d'agrément obtenus entre la scalimétrie et l'otolimétrie (94,9\% à 98,9\%) correspondent aux limites de fiabilité de la première méthode (SYCH, 1967 ; JENSEN, 1968). Les divergences les plus grandes semblent apparaître pour les poissons ayant passé plus d'une saison de croissance en mer ; mais l'échantillon très faible de ce type de poisson (3) ne permet pas de conclure. Le choix d'utiliser l'une ou l'autre des techniques est relié à l'espèce et dans le cas des salmonidés au taux de croissance. Ceci est parfaitement démontré par JONSSON (1985) qui utilise les otolithes pour déterminer l'âge de la truite comme sédentaire (juvéniles et géniteurs) et les écailles pour la truite de mer du même bassin (smolts et géniteurs) car celle-cia un taux de croissance plus élevé. 


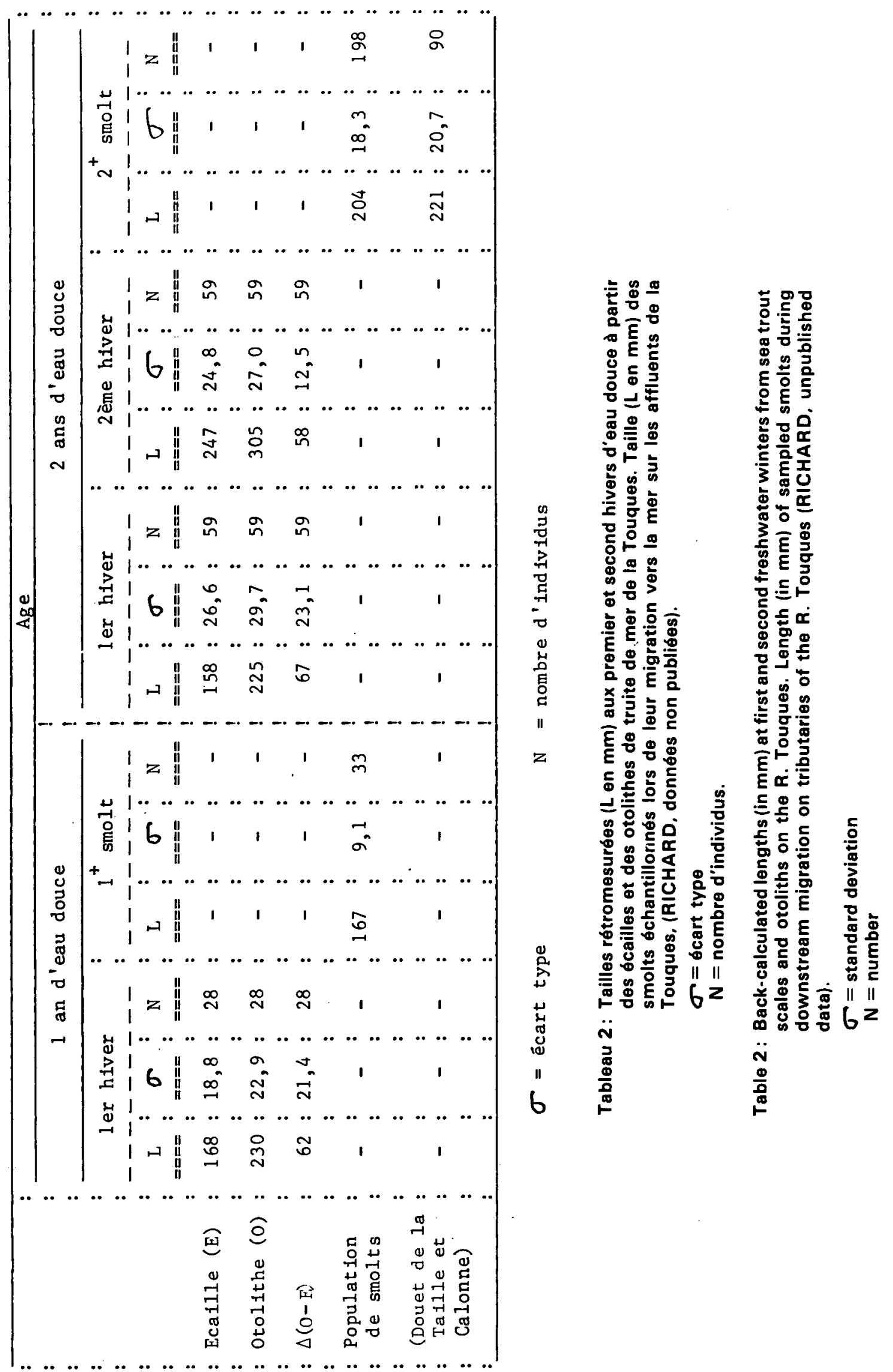




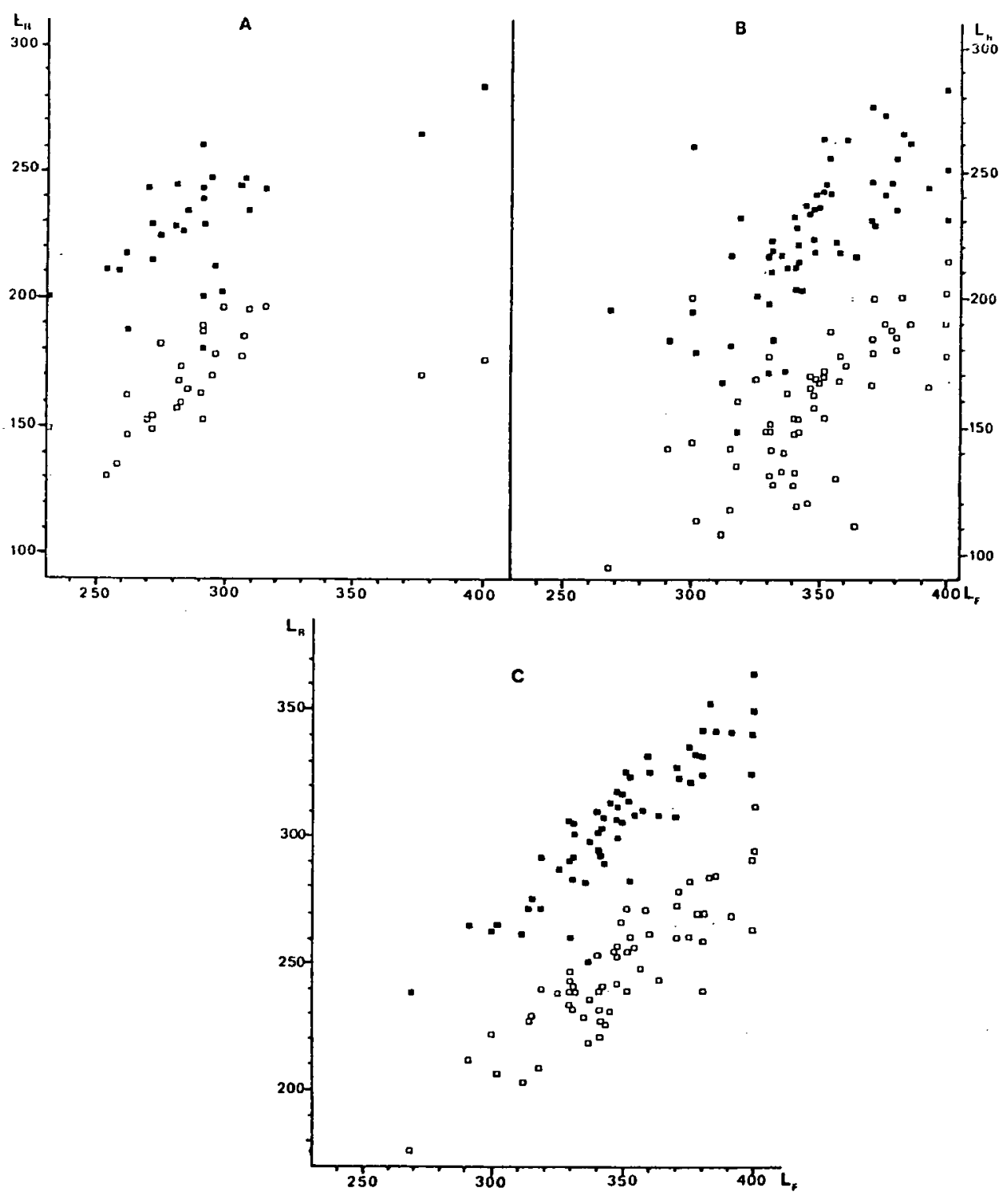

Figure 2: Relation entre les rétromesures effectuées aux premiers et second hivers d'eau douce a partir des écailles ( $\square$ ) et des otolithes ( $\square$ ) chez la truite de mer.
A - Longueur rétromesurée, au premier hiver pour les poissons âgés de 1 an d'eau douce.
B - Longueur rétromesurée au premier hiver pour les poissons âgés de 2 ans d'eau douce.
C - Longueur rétromesurée au deuxième hiver d'eau douce pour les poissons âgés de 2 ans d'eau douce.
$L_{P}=$ Longueur rétromesurée en $\mathrm{mm}$.
$L_{F}=$ Longueur fourche en $\mathrm{mm}$.

Figure 2: Relation between back-calculated length at first and second freshwater winters from scales ( $\square$ ) and otoliths $(\square)$ in sea trout.
A - Back-calculated length at first winter for the one freshwater year old fishes.
B - Back-calculated length at first winter for the two freshwater years old fishes.
C - Back-calculated length at second winter for the two freshwater years old fishes.
$L_{R}=$ back-calculated length in $\mathbf{~ m m}$
$L_{F}=$ fork length in $\mathbf{m m}$. 


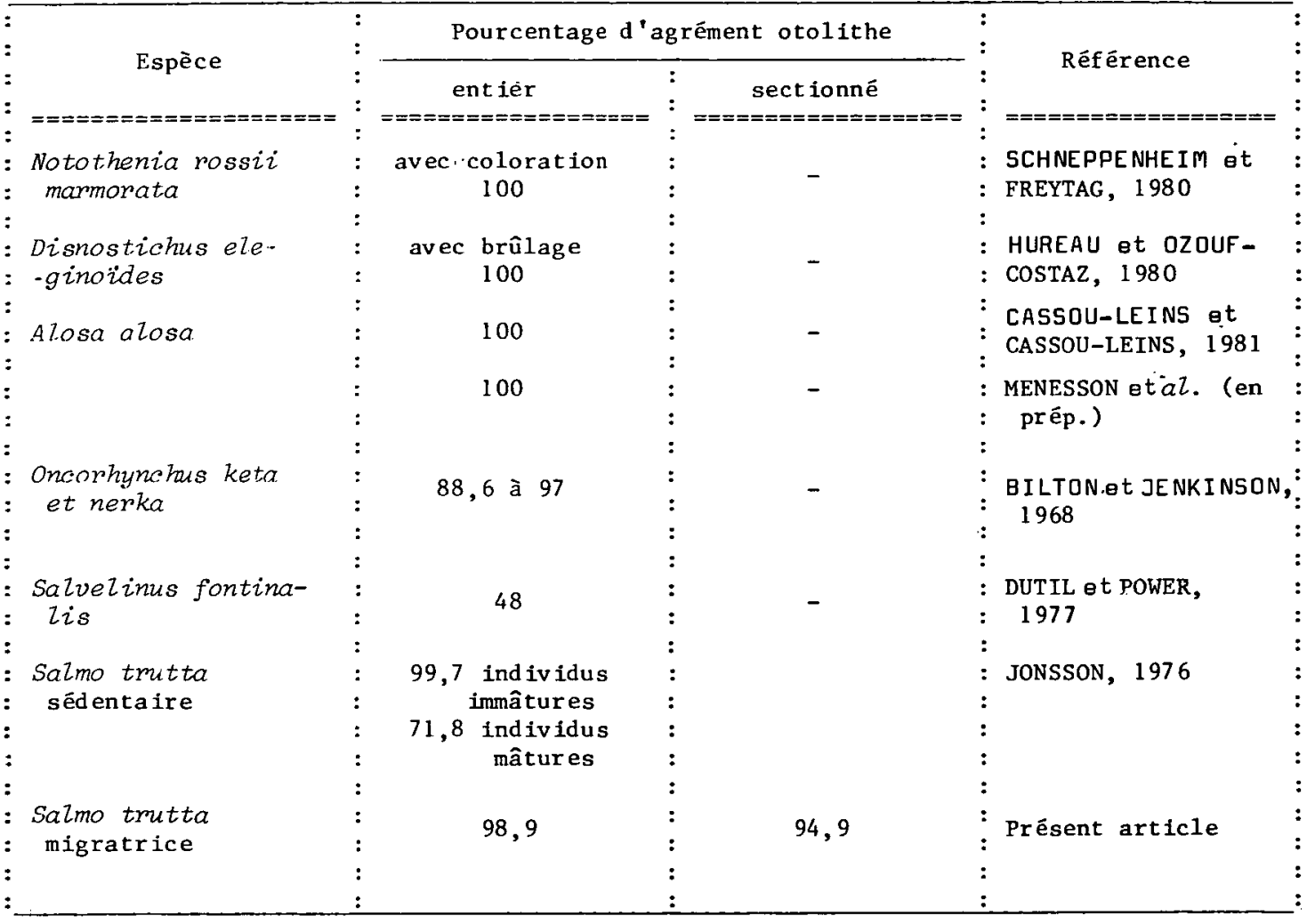

Tableau 3: Pourcentage d'agrément pour la détermination d'âge par scalimétrie et otolimétrie chez quelques espèces de poissons.

Table 3: Agreement percentage for age determination by scale and otolith reading in some species of fishes.

En otolimétrie, l'interprétation faite par lecture externe concorde plus avec celle réalisée par scalimétrie que lorsqu'est prise en compte la lecture interne (les pourcentages d'agrément ne sont cependant pas différents, $p<0,01)$. Pourtant, il est généralement reconnu que l'utilisation de sections d'otolithes améliore sa lisibilité et l'estimation de I'âge (PANNELLA 1974; BEDFORD, 1980 ; SOUPLET et DUFOUR, 1983 ; BURCHETT et al., 1984 ; CAMPANA, 1984 ; HOYER et al., 1985). Dans le cas de la truite de mer de la Touques, le seul désavantage de l'utilisation de l'otolithe entier reste le pourcentage important de non lisibilité.

La technique d'observation macroscopique de la structure des otolithes ne permet pas de distinguer de marques de fraie avant la migration marine et de reprise de croissance du smolt comme sur les écailles. Seuls de faux anneaux ont pu être observés à la fois sur ces derniers et sur les sections d'otolithes. La présence d'une large plaque opaque au centre de l'otolithe entier constatée également par DUTIL et POWER (1977): EVERSON (1980) et BURCHETT (1984) rend impossible cette observation. Par contre, l'analyse de la microstructure de l'otolithe au microscope à balayage électronique devrait permettre de mettre en évidence les marques de fraie (PANNELLA, 1971 et 1974 ; DENIEL, 1981) et la période de croissance en estuaire ou stade smolt (MARSHALL et PARKER, 1982 ; VOLK et al. 1985). Il est également certain que le niveau d'interprétation reste fonction de l'expérience acquise en otolimétrie.

Si le pourcentage d'agrément pour la détermination de l'âge est satisfaisant, il n'en est pas de même pour l'analyse de la croissance. L'écaille semble être la structure la plus fiable chez la truite de mer $\mathrm{O}^{+}$pour deux raisons :

- les longueurs rétromesurées à la fin des deux hivers d'eau douce sont très proches des tailles réelles des smolts échantillonnés sur la Touques (Tableau 2);

- l'ajustement longueur fourche - longueur de l'écaille présente une variance résiduelle plus faible et un coefficient de corrélation plus fort que l'ajustement réalisé à partir des otolithes. Pour ces derniers, une relation de type puissánce a également été testée mais donnait des valeurs rétromesurées supérieures à celles calculées par le modèle linéaire. 


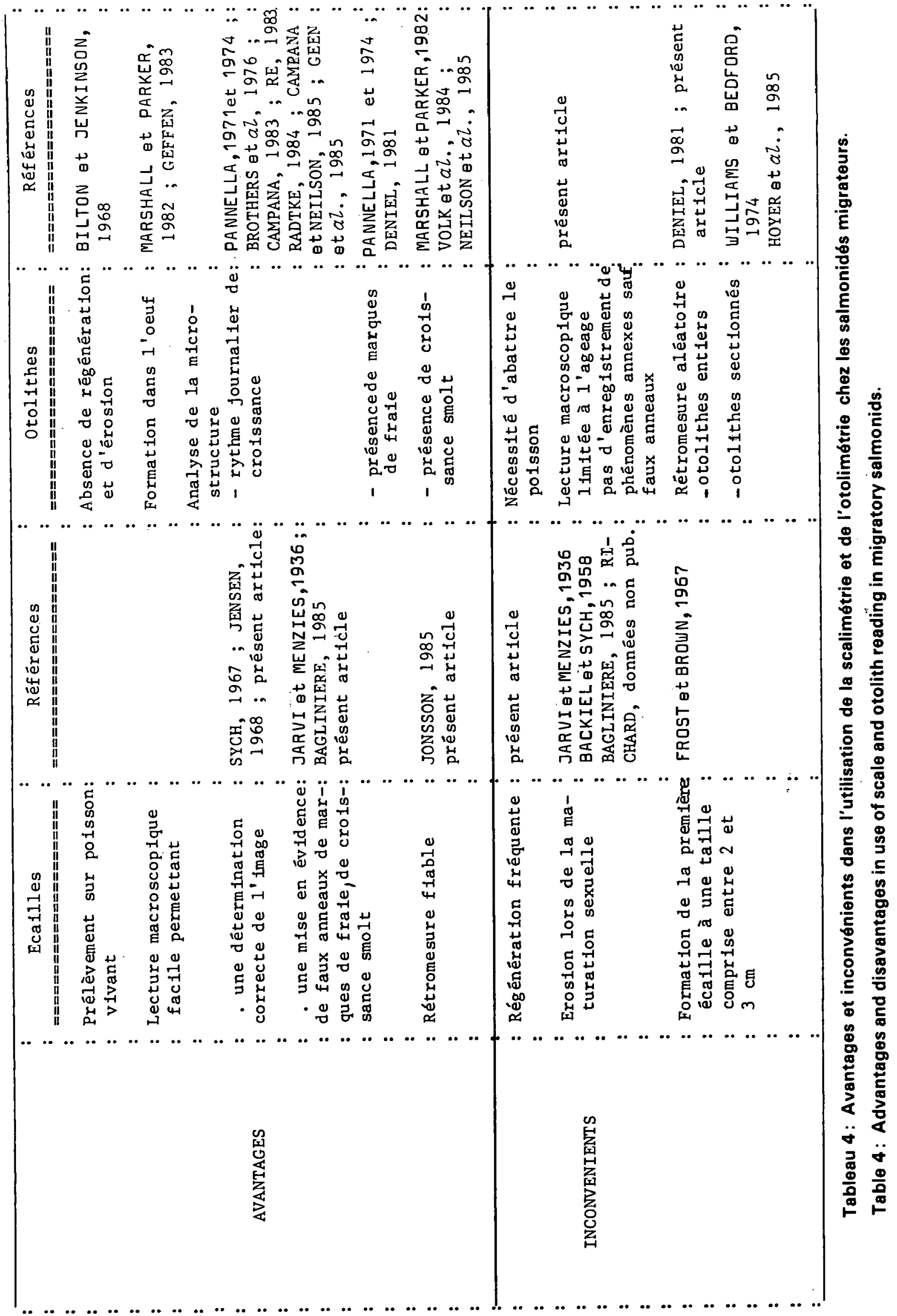


Sur la truite commune en Norvège, JONSSON et STENSETH (1977) considèrent que la structure la plus fiable pour analyser la croissance est l'otolithe. Dans leur étude sur la grande alose (Alosa alosa) de la Garonne, CASSOU-LEINS et CASSOU-LEINS (1981) ne trouvent pas de différence entre les tailles rétromesurées par les deux méthodes. Dans le cas de la truite de mer de la Touques, les valeurs rétromesurées à partir des otolithes sont toujours supérieures à celles estimées à partir des écailles et quel que soit l'âge, l'écart moyen reste relativement constant. Cela signifie que les différences peuvent provenir :

- de la difficulté à positionner le nucleus (DENIEL, 1981 ; WILSON et LARKIN, 1982) et la fin de I'hiver sur un otolithe alors que ceci est relativement facile sur une écaille;

- mais surtout du développement de l'otolithe qui se fait d'une manière non isométrique dans trois dimensions (PANNELLA, 1974). Ainsi le choix du rayon utilisé pour l'otolithe peut jouer un rôle (JONNSON et STENSETH, 1977). Il aurait été peut-être plus intéressant d'analyser la croissance pondérale comme dans l'étude effectuée par WILSON et LARKIN (1982). Enfin, l'utilisation d'une section d'otolithe passant par le nucleus (la structure analysée devient plane) devrait permettre d'obtenir des valeurs de croissance plus proches de la réalité (CAMPANA, 1984 ; BERG, 1985).

Les résultats obtenus dans cette étude et leur discussion permettent d'établir un tableau comparatif des avantages et des inconvénients dans l'utilisation des méthodes scalimétrique et otolimétrique pour la détermination de l'âge et de la croissance chez les salmonidés (Tableau 4). II en ressort que :

- la scalimétrie reste une méthode fiable couramment et facilement utilisable dans les études de dynamique en dépit d'une régénération assez fréquente (Tableau 5) et d'une érosion importante des écailles en période de fraie :

\begin{tabular}{|c|c|c|c|c|c|c|c|c|c|c|}
\hline ： & & $:$ & \multicolumn{3}{|c|}{ Nombre d'écailles par lame } & \multirow[t]{2}{*}{$\begin{array}{l}\text { : } \\
\text { : }\end{array}$} & Pourcentage $\mathrm{d}$ & & \multicolumn{2}{|c|}{ régēnération } \\
\hline * & & $:$ & Totales & : & Régénérées & & Moyen & : & ExtI & êmes \\
\hline : & & $:$ & $===== \pm=====-=$ & $:$ & 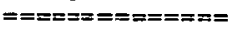 & : & $===\approx========$ & : & $======$ & $======$ \\
\hline : & & : & & : & & : & & : & & \\
\hline ; & Nombre moyen & $:$ & 39 & : & 24 & : & 61,5 & : & 7,7 & -100 \\
\hline 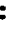 & & $:$ & & : & & : & & : & & \\
\hline 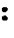 & Ecart-type & $:$ & 14,7 & : & 11,8 & : & - & : & & - \\
\hline : & & : & & : & & : & & : & & \\
\hline & $\begin{array}{l}\text { Nombre de poissons } \\
\text { ëchantillonnés }\end{array}$ & $:$ & 131 & $:$ & 131 & 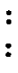 & - & : & & - \\
\hline & & $:$ & & : & & : & & : & & \\
\hline
\end{tabular}

Tableau 5 : Pourcentage (\%) d'écailles régénérées dans l'échantillon de truite de mer de la Touques.

Table 5 : Percentage of regenerated scales in sea trout sample of the R. Touques.

- l'otolimétrie reste avant tout une méthode lourde, peu utilisée et nécessitant l'abattage des individus. Cependant, les otolithes ne présentent pas de phénomène de régénération et de résorption. De plus, l'analyse de leur micro-structure apporte des données plus précises sur les interactions poisson-environnement que celle de la structure des écailles. Mais de telles analyses ne sont intéressantes que pour la compréhension de phénomènes particuliers.

\section{V - CONCLUSION}

Cette première étude comparative de détermination d'âge par les méthodes scalimétriques et otolimétriques (lectures externe et interne) chez la: truite de mer-de la Touques donne des résultats intéressants puisque le pourcentage d'agrément est toujours supérieur à $93 \%$. Les tailles rétromesurées à partir des otolithes sont toujours supérieures à celles estimées par les écailles qui sont proches des valeurs réelles observées. Ainsi l'écaille reste la structure la plus fiable et d'utilisation la plus simple pour la détermination de l'âge et de la croissance de la truite de mer d'une seule saison de croissance en mer.

Cependant, cette étude a comparé presque exclusivement les âges d'eau douce puisque les individus sont en quasi-totalité des $\mathrm{O}^{+}$de mer.

Ainsi à l'avenir, il apparaît intéressant de poursuivre une telle étude en améliorant la technique otolimétrique (niveau d'interprétation, observation de la microstructure) et en élargissant la compa- 
raison aux autres groupes d'âge de mer des salmonidés migrateurs (truite de mer et saumon atlantique). L'érosion générale des écailles de ces poissons au moment de la maturation sexuelle limite l'utilisation de la méthode scalimétrique pour la détermination de l'âge marin. L'absence d'érosion au niveau des otolithes doit permettre dans ce cas d'utiliser la technique otolimétrique en complément de la première sur les poissons morts après la fraie. Néanmoins, pour valider ces deux méthdodes de détermination d'âge et approfondir les relations âge-taille-structure osseuse, des recherches plus poussées doivent être envisagées en utilisant notamment des techniques d'osteochronologie (MEUNIER et al., 1979).

\section{REMERCIEMENTS}

Nous remercions le laboratoire IFREMER de Lorient et également celui de Sciences du Sol, INRA, de Rennes pour la préparation des coupes d'otolithes.

\section{REFERENCES}

BACKIEL T., SYCH R., 1958. Scales resorption and spawning marks in sea trout (Salmo trutta L.) from polish waters. Roczniki nauk. Rolnizych., 73: 119-148.

BAGLINIĖRE J.L., 1985. La détermination de l'âge par scalimétrie chez le saumon atlantique (SaImo salar): utilisation pratique et limites de la méthode. Bull. Fr. Pisc., 298 : 65-107.

BAGLINIERE J.L., MAISSE G., 1985. Precocious maturation and smoltification in wild atlantic salmon in the Armorican Massif, France. Aquaculture, $45: 249-263$.

BEALL E., 1979. Analyse scalimétrique d'une population de truites de mer (Sa/mo trutta L.) des lles Kerguelen, T.A.A.F., structure d'âge, croissance, reproduction. Thèse de troisième cyc/e, Univ. Paul Sabatier, Toulouse, 1 vol. 161 pp.

BEDFORD B., 1980. A method for preparing thin sections of large numbers of otolith embedded in black polyester resin. Cybium, 3ème série, $8: 6$.

BERG R., 1985. Age determination of eels, Anguilla anguilla L. : comparison of field data with otolith ring patterns. J. Fish. Biol., $26: 537-544$.

BILTON H.T., JENKINSON D.W., 1968. Comparison of the otolith and scale methods for aging Sockeye (Oncorhynchus nerka) and Chum (Oncorhynchusketa). J. Fish Res. Bd Canada, 25: 1067-1069.

BROTHERS E.B., MATHEWS C.P., LASKER R., 1976. Daily growth increments in otolith from larval and adult fishes. Fish. Bull. $74: 1-8$

BURCHETT M.S., 1984. Age determination of Notothenia rossiifrom South Georgia (Antarctica) using scales and otoliths. Cybium, 8 19-28.

BURCHETT M.S., DEVRIES A., BRIGGS A.J., 1984. Age determination and growth of Dissostichus mawsoni(Norman, 1937)(Pisces, Nototheniidae) from Mc Murdo sound (Antarctica). Cybium, 8 : 27-31.

CAMPANA S.E., 1983. Calcium deposition and otolith check formation during periods of stress in coho salmon, Oncorhynchus kisutch. Comp. Biochem. Physiol, 75A : 215-220.

CAMPANA S.E., 1984. Comparison of age determination methods for the starry flounder. Trans. Am. Fish. Soc., 113: 365-369.

CAMPANA S.E., NEILSON J.E., 1985. Microstructure of otoliths. Can. J. Fish. Aquat. Sci., 42 : 1014-1032.

CASSOU-LEINS F., CASSOU-LEINS J.J., 1981. Recherche sur la biologie et l'halieutique des migrateurs de la Garonne et principalement de l'alose Alosa alosa Linné. Thèse 3ème cycle, Inst. Nat. Polytechn. Toulouse, 1 vol. 392 pp.

DENIEL C., 1981. Les poissons plats (téléostéens, pleuronectiformes) en Baie de Douarnenez. Reproduction, croissance et migration des Bothidae, Scophthalmidae, Pleuronectidae et Soleidae. These d'Etat Fac. Sci. Techn. Univ. Brest, 1 vol. 476 pp.

DUTIL J.D., POWER G., 1977. Validité de la lecture des otolithes comparée à celle de la lecture des écailles pour la détermination de l'âge de l'omble de fontaine (Salvelinus fontinalis). Naturaliste Can., $104: 361-367$.

EVERSONI., 1980. Méthodes de détermination de l'âge chez les poissons antarctiques. Cybium, 3ème série, $11: 41-59$.

FROST W.E., BROWN M.E., 1967. The trout: Ed. Collins, London, 1 vol. 236 pp.

GEEN G.H., NEILSON J.D., BRADFORD M., 1985. Effects of $\mathrm{pH}$ on the early development and growth and otolith microstructure of Chinook Salmon (Oncorhynchus tschawytscha). Can. J. Zool., $63: 22-27$. 
GEFFEN A.J., 1983. The deposition of otolith rings in Atlantic salmon Salmo salar L., embryos. J. Fish. Biol, $23: 467-474$.

HOYER M.V.. SHIREMAN J.V., MACEINA M.J., 1985. Use of otoliths to determine age and growth of largemouth bass in Florida. Trans. Am. Fish. Soc., 114: 307-309.

HUREAU J.C., OZOUF-COSTAZ C., 1980. Age determination and growth of Dissostichus eleginoides Smitt, 1898, from Kerguelen and Crozet Islands. Cybium 3ème série, 8 : 23-32.

JARVIT.H., MENZIES N.J.M., 1936. The interpretation of the zones on scales of salmon, sea trout and brown trout. Cons. perm. expl. mer, XCVII, 1 vol. 63 pp.

JENSEN K., 1968. Sea trout (Salmo trutta L.) of the River Istra, Western Norway. Rep. Inst. Freshwat. Res. Drottningholm. $48: 187-213$.

JONSSON B., 1976. Comparison of scales and otoliths for age determination in brown trout, Salmo trutta L.. Now. J. Zool., 24 : 295-301.

JONSSON B., 1985. Life history patterns of freshwater resident and sea-run migrant brown trout in Norway. Trans. Am. Fish., Soc., 114: 182-194.

JONSSON B., STENSETH N. CHR., 1977. A method for estimating fish length from otolith size. Inst. Freshwat. Res. Drottningholm Rep. $56: 81-86$.

LECREN E.D., 1947. The determination of the age and growth of the perch (Perca fluviatilis) from the opercular bone. J. Anim. Ecol.. $16:$ 188-204.

MAC PHAIL D.K., 1974. Photographic reproductions of Atlantic salmon scales from fish of known sea ages. Techn. Rep. Series no MAR/X-74-1, 1 vol. 55 p.

MAISSE G., BAGLINIERE J.L., 1983. Mise en évidence d'une marque de fraie dans la phase eau douce des écailles de saumon atlantique du Massif Armoricain. I.C.E.S. CM 1983/M 28: 1 -7.

MARSHALL S.L., PARKER S.S., 1982. Pattern identification in the microstructure of sockeye salmon (Oncorhynchus nerka) otoliths. Can. J. Fish. Aquat. Sci., 39: 542-547.

MEUNIER F.J., PASCAL M., LOUBENS G., 1979. Comparaison de méthodes squelettochronologiques et considérations fonctionnelles sur le tissu osseux acellulaire d'un osteichthyen du lagon néo-calédonien, Lethrinus neubulosus (Forskall, 1775). Aquaculture, 17 : 137-157.

NEILSON J.D., GEEN G.H., BOTTOM D., 1985. Estuarine growth of juvenile chinook salmon (Oncorhynchus tschawytscha) as inferred of from otolith microstructure. Can. J. Fish. Aquat. Sci., 42 : 899-908.

PANNELLA G., 1971. Fish otoliths : daily growth layers and periodical patterns. Science, 173 : $1124-$ 1127.

PANNELLA G., 1974. Otolith growth patterns : an aid in age determination in temperate and tropical fishes. In TB BAGENAL (editor), the ageing of fish, 1973 symposium, Unwin Brothers, p. 28-39.

PIPPY J., REDDIN D., 1982. Report on the 1981/82 International Atlantic Salmon Scale Reading Exercise. ICES CM 1982/M:14, 2 pp.

RADTKE R.L., 1984. Formation and structural composition of larval striped Mullet otoliths. Trans. Am. Fish. Soc., 113: 186-191.

RE P., 1983. Daily growth increments in the sagitta of pilchard larvae Sardina pilchardus (Walbaum, 1972) (Pisces :Clupeidae), Cybium, 7:9-15.

RICHARD A., 1981. Observations préliminaires sur les populations de truite de mer (Salmo trutta L.) en Basse-Normandie. Bull. Fr. Piscic., 283: 114-124.

SCHNEPPENHEIM R., FREYTAG G., 1980. Age determination by staining otoliths of Notothenia rossii marmorata with ninhydrin. Cybium, 3ème série. 8: 13-15.

SOUPLET A., DUFOUR J.L., 1983. Développement des techniques de lecture des otolithes en coupes fines. Rapp. Techn. ISTMP, 5 - 5 pp.

SYCH R., 1967. Confidence estimation of a fish age determination from scales as exemplified by sea-trout (Salmo trutta L.). Roczniki nauk Rolnizych., 90: 281-303.

VOLK E.C., WISSMAR R.C., SIMENSTAD C.A., EGGERS D.M., 1984. Relationship between otolith microstructure and the growth of juvenile salmon (Oncorhynchus keta) under different prey rations. Can. J. Fish. Aquat. Sci., 41 : 126-133.

WILLIAMS T., BEDFORD B.C., 1974. The use of otoliths for age determination. In T.B. BAGENAL (editor). The ageing in fish, 1973 Symposium, Unwin Brothers, p. 114-123.

WILSON K.H., LARKIN P.A., 1982. Relationship between thickness of daily growth increments in sagittae and change in body weight of sockeye salmon (Oncorhynchus rierka) fry. Can. J. Fish. Aquat. Sci., $39:$ 1335-1339. 